\title{
Fifth Gen Control for Industrial Wet Grinders
}

\author{
Meenakshi Subramanian ${ }^{1}$, Dr.B.Sargunam ${ }^{2}$, Akalya.A ${ }^{3}$, Deshika.P ${ }^{4}$, \\ Department of Electronics and Communication Engineering ${ }^{[1,3,4]}$, \\ Associate Professor ${ }^{[2]}$, \\ School of Engineering, Avinashilingam Institute for Home Science and Higher Education for Women, \\ Coimbatore, India.
}

\begin{abstract}
A number of conventional methods have been proposed till date, but the proposed model creeps in a new set of innovative notions for wet grinders incorporating technical stuff into existence. The proposed model quenches the intension to digitalise the hand operated traditional methods available for wet grinders. Grinding is a labour intensive and manpower-oriented work. This is a real time consumer electronic problem. The proposed model is designed with smart facilities like speed regulation, time regulation, temperature regulation and selection mode. This model helps to reduce the work burden of the user and any unskilled user can make use of this technology easily. The main goal of this smart wet grinder control is to improve the efficiency of grinding with continuous monitoring and extend it to the field of commercial electronics.
\end{abstract}

Keywords - Quarter hp motor, Variable Frequency Drive (VFD), Touch panel, PIC microcontroller, LCD display

\section{INTRODUCTION}

The wet grinder is a primary mode of grinding raw food materials. There are many types of grinders. Initially the grinding was initiated manually by user. It has been used for domestic and commercial purposes to do coarse and wet grinding. The main disadvantage of this type of grinder is, it does not have any facility for availing input from user.

Wet grinder is a household appliance used for preparing batter, out of which some materials are prepared. Rice and dhal are the main raw materials used in the preparation of the batter. Before the invention of the mechanical wet grinders, hand operated grinding stones were used for making this batter. After the invention of mechanical wet grinders, the preparation of batter is in quick manner than the traditional grinding. But the energy consumption of these mechanical wet grinders is high and requires manual checking of flour every time for the grinding time and speed for rice and dhal are not same so manual checking is needed.

Sidiq Syamsul Hidayat, proposed the concept of application of Wireless Sensor Network (WSN) technology in the implementation of the temperature and humidity monitoring system on paddy drying machine. The advantage of the WSN in a dryer is the number of sensors can be increased without increasing the number of cable installation on the machine. [1]

Shuping Chen et al., proposed a thesis on controlling system of the grinding based on multi sensors and aims at machining some super rigid materials such as ruby, sapphire and diamond. This method with the help of the nature of positioning, details the shape and posture of a position in machining, and a smart grinding force sensor is made up; the poses are detected by multi optical grating sensors. [2]
Tao Yang et al., proposed a concept on user's interactive control venture and data architecture design. From the user experience an interactive interface design principles and quench to design a simple, easy to use, stable performing, multi - oriented interactive interface was the main feature in this paper . [3]

L. Dudás, proposed a key point on grinding wheel surfaces for grinding the rotor of special type pumps, compressors and expansion machines that have only rotary parts. For the surface generation a specialized CAD system is used that is originally intended for the innovation of gears. [4]

Li Penghui et al., proposed a concept on grinding processes in resource processing plants which are featurised by non-linearity, time-variability, along with large inertia. However, their limiting conditions change during operation, and technical features such as grinding particle size are difficult to measure and optimizing control is difficult to achieve. [5]

Yue Feng et al., proposed a concept on the structure and cooling issues of grinding high speed motorized spindle. The device runs at a speed via PLC frequency. According to the basic functions of Distributed Control System, the corresponding software is developed.[6]

Mingli Xie et al., proposed a concept on special characteristics of grinding, including a selection of inspection parameters, a model of grinding feed system, and the design of controller. Results of detailed analysis reveal that this system can ensure a stable grinding force and enhance the machining quality [7].

S. Ekram et al., proposed a concept on the design and development of a high efficiency 3-phase, 6/4 switched reluctance motor (SRM) for driving a $280 \mathrm{~W}$ mixer-grinder. The conventional motor under usage for this purpose up till now is a complex one and because of its limitations like the requirement of a commutator with brushes, and absence of easy controllability, the efficiency never exceeds 70\%. [8]

The techniques referred from these papers lags in efficiency and user friendliness in its operation. The proposed model has improved performance and simple in operation.

Variable frequency drive and quarter hp motor is one of the most important part of the system. VFD is an adaptable speed drive used in driver system to control speed of AC motor and torque by varying motor input frequency and voltage. Conventional grinders only have a mere motor and driver circuitry which can only control the grinders manually to some extent. 
Here the user needs to perform complex operations to complete his/her work such as settling up of the grinding, halting the process of grinding and check out for heavy loading and consistency of batter. Here the user must continuously monitor and respond accordingly. Lack of proper inspection may turn the batter or dough into a worser one and the required consistency may not be achieved. A better care and monitoring assistant are required to get the perfect batter.

Traditional methods proposed till date can only run on fixed speed and hence waste lots of valuable energy and time. We are here to implement a quality enhanced consumer electronics-based product to improvise automation and reduce man power. It uses user friendly algorithms based on user's decision.

The continuous monitoring of the factors like temperature, loading, consistency of the batter, speed is highly required and based on that the corrective or required measures needs to be applied. In contrary the existing system available are not easily accessible, affordable and does not have such robust facilities. To bring in such sophisticated features into the system, a driver and sensors are employed for the purpose of an early intimation which helps to reduce the work burden of the user operating the system. In the proposed smart model, an early warning is made available to the user via an alarm provisioned in the system.

\section{BLOCK DIAGRAM}

The Fig. 1 illustrates the block diagram of the proposed system.

A touch panel is a pointing device provisioned with a sensor along with a specialized surface that can translate the motion and position of the user's fingers to a relative hub on the system which is displayed as output on the screen. The module introduced operates based on the user's input. The grinder mainly uses touch panel to vary speed, time, menu and power input based on user's input. This grinder control mainly consists of microcontroller, touch panel, LCD display, and power supply. The user inputs are converted into machine understandable values. Often there exist situations where we need to urgently change the speed of grinding and time required for grinding at any instant during grinding.

In kitchen areas like hotels or hostels and other such busy locations the chef need not have to manually check in every time to find the status of grinding. Here the chef may set in the inputs at initial stage through the touch panel, the message is, then transmitted and displayed on the screen. To demonstrate this concept, an LCD screen is used here to display features mentioned above.

The LCD is interfaced with a PIC microcontroller to direct and regulate the sophisticated environment. A touch panel module is used to receive user inputs, send them to the microcontroller for converting them into machine understandable instruction and further into the process and execute it. The microcontroller then displays the information via the LCD screen.

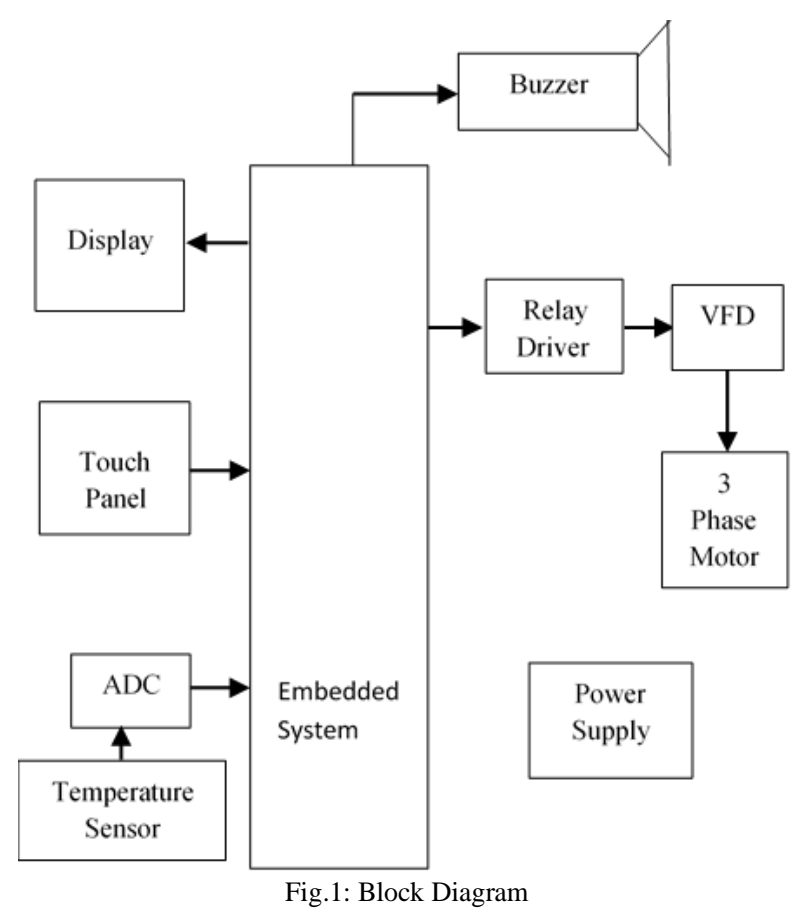

This grinder can be used in various places including hostels, schools, colleges, offices and hotels to grind effectively.

A touch panel or track panel is a pointing device featuring a tactile sensor, a specialized surface that can translate the motion and position of a user fingers to a relative position on the operating system that is made output on the LCD display.

A touch panel module is used to receive user inputs, send them to the microcontroller for converting them into machine understandable instruction and further into the process and execute it. The microcontroller then displays the message on the LCD screen.

Touch panels operate in several ways, including capacitive sensing and resistive touch screen. The most common technology used in the earlier days senses the change of capacitance where a finger touches the panel. Capacitance-based touch panels will not sense the tip of a pencil or other similar ungrounded or non-conducting element. On the other hand, fingers insulated by a glove may also be problematic.

While touch panels like touch screens, are able to sense absolute position, resolution is limited by their size. For common use as a pointer device, the dragging motion of a finger is translated into a finer, relative motion of the cursor on the output to the display on the operating system. Sometimes even hardware buttons equivalent to a standard mouse left and right buttons are position adjacent to the touch panel.

A timer is a property that provides us to perform a function at a particular time. The Display timer is a versatile display that functions as an Elapsed Timer or Pre-set Timer, with full-featured user programmability. These modes provide either a level active or edge triggered start/stop operation. Device counts down from a specified time interval.

Digital displays use technologies such as LCD to display menus. The mode of grinding shifts according to the user's menu choice. 
A Variable Frequency Drive is a classification of controller for motor which drives an electric motor by varying the frequency and voltage applied to the electric motor. Variable-frequency drive (VFD), is a module of adjustable-speed drive used in the grinder to control the speed and torque of motor by varying motor input frequency and voltage.

The VFD controller is a conversion system consisting of three different sub-systems such as rectifier bridge converter, a direct current (DC) link, and an inverter. Speed can also be controlled remotely and locally. The operating parameters of a VFD can be programmed via dedicated programming software. VFDs are capable of blocking most programming changes while running.

A temperature sensor is a device that provides temperature measurement with the help of an electrical signal. A thermocouple consists of two different metals that generate electric voltage in direct proportion to temperature variations. A Resistance Temperature Detector is a component that will change its electrical resistance in direct proportion to temperature changes.

\section{METHODOLOGY}

The basic methodology of the system we have proposed consists of the following modules in the user can set the parameters as per his/her choice. Here the first module consists of menu choice in which user has selector modes 0 , 1 and 2 for selecting Rice, Dal and Dough for grinding. 'Next module is timer unit in which user selects the time input for grinding in terms of seconds after the time ends the motor speed reduce in a gradual manner to zero(0) and the device stops running. Next module is power supply in which single phase AC $230 \mathrm{~V}, 50 \mathrm{~Hz}$ supply is given to bridge rectifier to give DC supply then it is passed through filter to smooth it out. Then an inverter is used to convert the DC to AC and given as input supply to motor and the motor runs according to set speed.

The following steps are performed to initiate the process of grinding:

\section{Step 1: Obtaining inputs from the end user}

The speed of grinding, the mode in which the grinding should happen, timer inputs and temperature threshold is set in by the user in this step.

\section{Step 2: Converting the input into machine understandable values}

The obtained speed from user is now converted into digitized values for machine inputs. The amiable inputs to machine are recognized. With the help of microcontroller unit the required input for the different components in the system are precisely applied.

Step 3: Varying power consumption via user inputs during the course of grinding

According to the need of end user grinding requirements power required for the motor to run is obtained.

Step 4: Applying machine understandable values to motor speed inputs (VFD)

According to the users input the frequency applied to the drive changes and accordingly its output voltage differs. The differed output voltage based on user's choices at any instant of grinding is then applied to the grinder.

\section{Step 5: Controlling the operation via microcontroller}

It acts as the heart of the entire system controlling various operations according to various time slots. It works on the priority of the available tasks. If the present task is completed it shifts to next task. The main objective here is to run the motor and to run the wet grinder for required time.

Step 6: Making user inputs active during entire duration:

If user wants to change any of the parameters (in case), they are allowed to change at any moment. Change motor speed at any instant of grinding and repeat above mentioned steps.

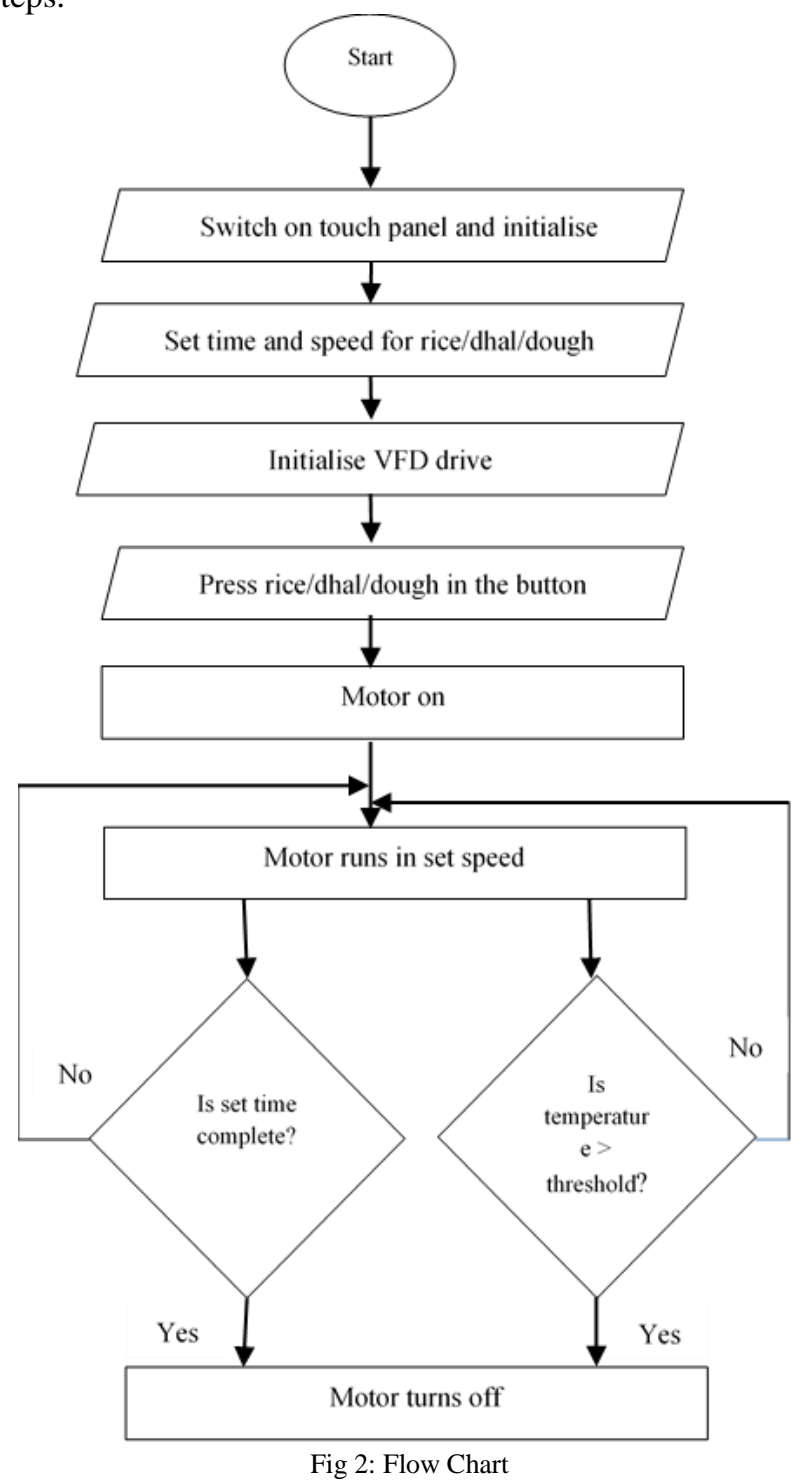

\section{Step 7: Miscellaneous:}

During the course of grinding if any temperature hike takes place. It is indicated via a buzzer. After the time set in is completed the grinder intimates the user via the buzzer that the grinding is completed and the batter is ready to use. Followed by this the user can collect the prepared batter grinded as per the user's choice.

The buzzer is used to indicate that the grinding is completed and also used to indicate the insufficiency of water.

The process is clearly shown in the flowchart in Fig. 2. 


\section{RESULTS}

The model circuit for smart control for wet grinders has been simulated and tested using MATLAB software. The hardware for smart control circuit for industrial wet grinder is implemented and tested for controlling the motor. The test has been carried out for different conditions and found to be working. The grinder is tested with all the three grains, for a particular temperature threshold and for a particular set time.

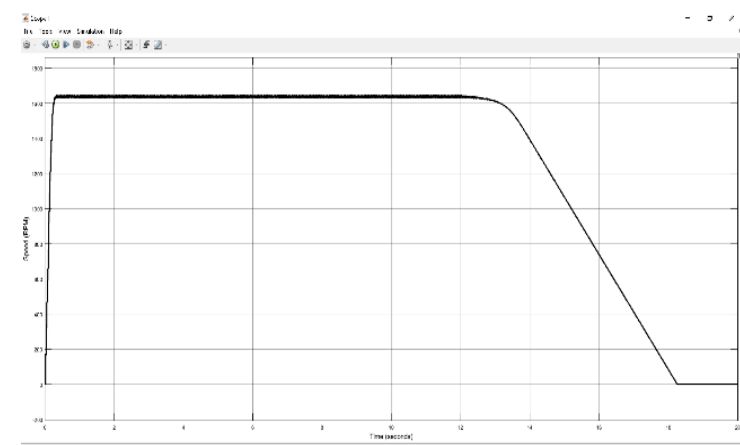

Fig. 3: Output for grinding Rice within temperature threshold

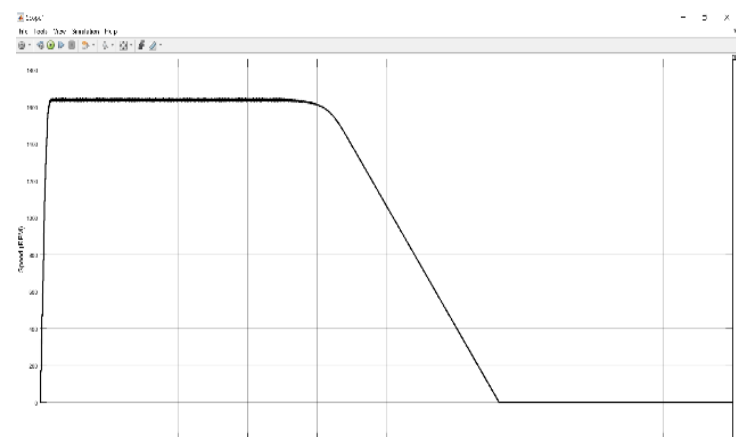

Fig. 4: Output for grinding rice greater than temperature threshold

The graph shown in Fig. 3 and 4 depicts the condition for grinding rice less than and greater than temperature threshold respectively. Here the parameter time is taken on $\mathrm{x}$-axis and speed on y-axis.

When the system is running under the threshold temperature, it runs for the time set by the user. If the temperature increases more than the threshold temperature set by the user, immediately the speed of the motor is gradually reduced and finally the motor is stopped.

Under menu choice rice is selected by user under this mode. According to the user's choice the speed is set in via a VFD drive. The temperature threshold is set in by the user and from the system the temperature is continuously observed. As shown in Fig. 3 if the system temperature is below the threshold set the system runs till the end of set time and stops gradually. When the system temperature is above threshold, the grinder ceases to run before the grinding time set by the user as shown in Fig. 4. The system stops in a gradual manner via the circuit breaker.

The snapshot of the display board during the initialisation is shown in Fig. 5. The parameters displayed in the display board during the grinding is shown in Fig. 6.

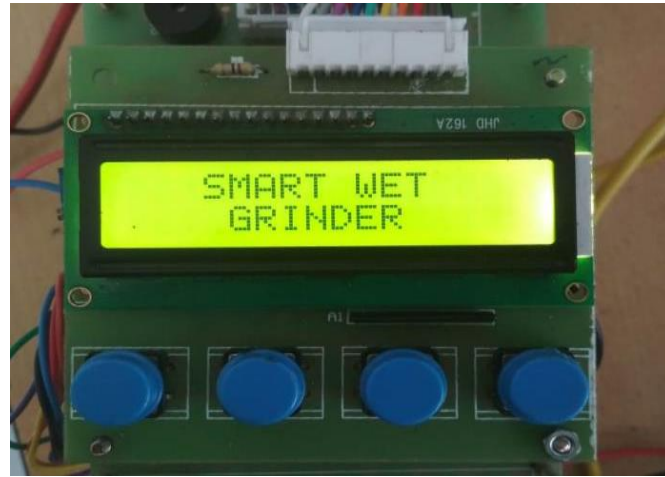

Fig. 5: Snapshot of Display board during Initialisation

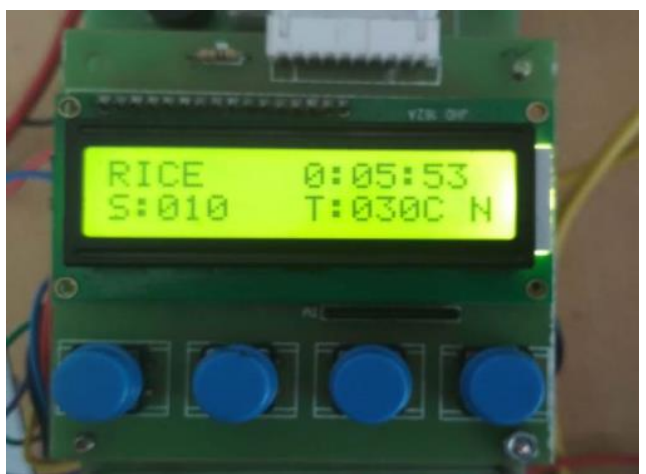

Fig. 5: Snapshot of Display board during Grinding

\section{CONCLUSION}

In this paper the smart control for industrial wet grinder has been developed to ease out the work burden of user. This will continuously monitor the parameters of grinding such as speed and temperature. Once the temperature level reaches the threshold value it will give alert to the user through the buzzer and LCD display. The temperature hike and time left to grind will be displayed in LCD. It is implemented to improve the manpower-oriented and time consuming job of the users. This system is flexible and cost effective and monitors all the parameters in a contiguous manner.

This system is found to be compact, user friendly and less complex which can be readily used in order to perform the grinding operation. This can be implemented and used in hotels, hostels, canteens and mess.

\section{FUTURE SCOPE}

Auto cleanser can be implemented in the grinder. This helps the users to set the mode of cleaning which in turn cleanses the entire system and provides a neat surface for future use. Then speed of grinding can be made limitless as per the user's choice without having any constraints. Child lock can be implemented into the system if required when it is implemented for domestic purpose.

\section{REFERENCES}

[1] Hidayat, S. S., Suharjono, A., Prasetyo, T., Jumi, Roihatin, A., Alfauzi,A. S., "Development of Two-In-One Type Drying Machine and Grinder Control System Based On Wireless Sensors Network", International Conference on Applied Science and Technology,(2018).

[2] Chen, X., Li, L., Wu, Q., "Effects of abrasive grit shape on grinding performance", 23rd International Conference on Automation and Computing (ICAC). doi:10.23919/iconic. 2017. 8081983, (2017) 
[3] Yang, T., Shanda, W., Gao, X., Li, J., Zhu, Y., Dai, F., "Research on the Robot Grinding and Polishing Production Line Interface Design Based on Theory of AD", 9th International Symposium on Computational Intelligence and Design (ISCID). doi:10.1109/iscid.2016.1014, (2016).

[4] Dudas, L., "Design of nonconventional grinding wheels with specialized CAD system", 4th IEEE International Symposium on Logistics and Industrial Informatics. doi:10.1109/lindi.2012.6319503, (2012).

[5] Li Penghui, Wang Jianmin., "Automatic control system of grinding process", 6th International Forum on Strategic Technology. doi:10.1109/ifost.2011.6021045, (2011).

[6] Feng, Y., \&amp; Zhao, Y., "Research on grinding spindle frequency distributed control system", International Conference on Electronic Mechanical Engineering and Information Technology. (2011)

[7] Xie, M., Hu, Z., Zhang, Y., "Research on Adaptive Control of Grinding Force of CNC Cam Grinder", International Workshop on Intelligent Systems and Applications. doi:10.1109/iwisa.2009.5073054, (2009).

[8] Ekram, S., Ravi, N., Rajagopal, K. R., Mahajan, D., "Design and Development of a High Efficiency Switched Reluctance Motor for a Mixer-Grinder Application", 33rd Annual Conference of the IEEE Industrial Electronics Society, (2007). 